\title{
Topological classification of gapped spin chains: Quantized Berry phase as a local order parameter
}

\author{
T. Hirano, ${ }^{1, *}$ H. Katsura, ${ }^{1, \dagger}$ and Y. Hatsugai ${ }^{1,2, \sharp}$ \\ ${ }^{1}$ Department of Applied Physics, The University of Tokyo, 7-3-1 Hongo, Bunkyo-ku, Tokyo 113-8656, Japan \\ ${ }^{2}$ Institute of Physics, University of Tsukuba, 1-1-1 Tennodai, Tsukuba, Ibaraki 305-8571, Japan \\ (Received 9 January 2008; revised manuscript received 18 January 2008; published 26 March 2008)
}

\begin{abstract}
We characterize several phases of gapped spin systems by local order parameters defined by quantized Berry phases [Y. Hatsugai, J. Phys. Soc. Jpn. 75, 123601 (2006)]. This characterization is topologically stable against any small perturbation as long as the energy gap remains finite. The models we pick up are $S=1,2$ dimerized Heisenberg chains and $S=2$ Heisenberg chains with uniaxial single-ion-type anisotropy. Analytically, we also evaluate the topological local order parameters for the generalized Affleck-Kennedy-Lieb-Tasaki model. The relation between the present Berry phases and the fractionalization in the integer spin chains are discussed as well.
\end{abstract}

DOI: 10.1103/PhysRevB.77.094431

PACS number(s): 75.10.Jm, 03.65.Vf, 73.43.Nq, 75.10.Pq

\section{INTRODUCTION}

Characterizing quantum many-body systems is one of the important topics in condensed matter physics. The GinzburgLandau (GL) theory has been quite successful to describe many phases based on a concept of the symmetry breaking and the local order parameter. Despite its remarkable success, novel types of phases which are not well described by the (classical) local order parameters have been found in many systems. Concepts of topological order and quantum order are trial to overcome the difficulties of the classical GL theory with the symmetry breaking. ${ }^{2-4}$ One of the characteristic features of the topological insulators is that localized states, such as the edge states, appear near the system boundaries, even though the system without boundaries has a finite energy gap. Examples of such systems are quantum Hall liquids, ${ }^{5-7}$ Haldane spin systems, ${ }^{8,9}$ polyacetylene, ${ }^{10}$ and spin-Peierls system. ${ }^{11}$ Recently, it has become clear that the bulk-edge correspondence ${ }^{6,7}$ has an intimate relation to the entanglement entropy, ${ }^{12-14}$ which has been discussed to detect nontrivial structures of topologically ordered states. ${ }^{13-16}$

Recently, one of the authors proposed to use another quantum quantity, quantized Berry phases,${ }^{17}$ to define a topological local order parameter. ${ }^{1,3,4}$ One can define a topological local order parameter by the Berry phases even though there is no classical order parameter. The Berry phases are a typical quantum quantity based on the Berry connection which is defined by the overlap between the two states with infinitesimal difference. It implies that the topological local order parameter defined in Ref. 1 is a quantum order parameter that does not have any corresponding classical analogs. Further, it has a conceptual advantage for the topologically ordered phases, since it is quantized to 0 or $\pi$ $(\bmod 2 \pi)$ when the ground state is invariant under some antiunitary transformation. It implies a topological stability that the quantized Berry phase does not change against any small perturbation. The Berry phase is given by an integration of the Berry connection defined by the local $U(1)$ twist on a link of a lattice. Then, the quantum phases can be categorized by the texture pattern of the Berry phases $(0$ or $\pi$ ). It has been successfully applied to several gapped quantum systems. For example, the ground states of the $S=1 / 2$ dimerized Heisenberg models (in one and two dimensions even with frustrations) ${ }^{1}$ can be characterized by the pattern of $\pi$ Berry phases on the bonds which indicate the locations of dimer singlets. In a case of the $t-J$ model,${ }^{18}$ it is characterized by the texture pattern of the non-Abelian Berry phase, which describes itinerant singlets. Also, for the $S=1$ Heisenberg model, its ground state as the Haldane phase was characterized by the uniform $\pi$ Berry phases. This topological order parameter also clearly describes a quantum phase transition between the Haldane phase and the large-D phases. ${ }^{19}$

In this paper, we calculate the topological local order parameter by the quantized Berry phase for several gapped quantum spin chains. There are substantial numbers of studies for the Haldane phase. ${ }^{20-23}$ Then, it has been clarified that the Haldane phase can be characterized by the hidden $Z_{2}$ $\times \mathbb{Z}_{2}$ symmetry breaking ${ }^{24,25}$ which describes a nonlocality of the Haldane phase (by the string order parameters). ${ }^{26-28}$ On the other hand, the topological order parameter by the Berry phases is local and quite useful for the $S=1$ case to describe the phase and the quantum phase transition. ${ }^{19}$ Here, we further investigate generic situations, such as the several Haldane phases in the dimerized $S=1,2$ Heisenberg chains $\mathrm{s}^{29,30}$ and the $S=2$ Heisenberg chain with uniaxial single-ion-type anisotropy. ${ }^{31-34}$ We also study the Berry phase of the generalized valence-bond-solid (VBS) state analytically and interpret the numerical results in terms of the reconstruction of the valence bonds.

\section{DEFINITION OF THE BERRY PHASE}

Let us start with defining the Berry phase in a quantum spin system. The Berry phase is defined when the Hamiltonian has parameters with periodicity assuming a finite energy gap between the ground state and the excited states. ${ }^{17}$ For the parameter dependent Hamiltonian $H(\phi)$, the Berry phase $\gamma$ of the ground state is defined as

$$
i \gamma=\int_{0}^{2 \pi} A(\phi) d \phi
$$

where $A(\phi)$ is the Abelian Berry connection obtained by the single-valued normalized ground state $|\mathrm{GS}(\phi)\rangle$ of $H(\phi)$ as 
$A(\phi)=\left\langle\mathrm{GS}(\phi)\left|\partial_{\phi}\right| \mathrm{GS}(\phi)\right\rangle$. This Berry phase is real and quantized to 0 or $\pi(\bmod 2 \pi)$ if the Hamiltonian $H(\phi)$ is invariant under the antiunitary operation $\Theta$, i.e., $[H(\phi), \Theta]=0^{3}$. Note that the Berry phase is "undefined" if the gap between the ground state and the excited states vanishes while varying the parameter $\phi$. We use a local spin twist on a link as a generic parameter in the definition of the Berry phase. ${ }^{1}$ Under this local spin twist, the following term $S_{i}^{+} S_{j}^{-}+S_{i}^{-} S_{j}^{+}$in the Hamiltonian is replaced with $e^{i \phi} S_{i}^{+} S_{j}^{-}+e^{-i \phi} S_{i}^{-} S_{j}^{+}$, where $S_{i}^{ \pm}=S_{i}^{x} \pm i S_{i}^{y}$. The Berry phase defined by the response to the local spin twists extracts a local structure of the quantum system. By this quantized Berry phase, one can define a link variable. Then, each link has one of the three labels: "0 bond," " $\pi$ bond," or undefined. It has a remarkable property that the Berry phase has topological robustness against the small perturbations unless the energy gap between the ground state and the excited states closes. On the other hand, the undefined indicates an existence of the quantum phase transition. In order to calculate the Berry phase numerically, we introduce a gauge-invariant Berry phase ${ }^{1,35}$ on a lattice. It is defined by discretizing the parameter space of $\phi$ into $N$ points as

$$
\gamma_{N}=-\sum_{n=1}^{N} \arg A_{N}\left(\phi_{n}\right), \quad \phi_{n}=\frac{2 \pi}{N} n,
$$

where $A_{N}\left(\phi_{n}\right)$ is defined by $A_{N}\left(\phi_{n}\right)=\left\langle\mathrm{GS}\left(\phi_{n}\right) \mid \mathrm{GS}\left(\phi_{n+1}\right)\right\rangle$, $\phi_{N+1}=\phi_{1}$. We expect $\gamma=\lim _{N \rightarrow \infty} \gamma_{N}$. To calculate $\gamma_{N}$, we use the Lanczos method to diagonalize the Hamiltonian in the subspace of $\sum_{i} S_{i}^{z}=0$.

\section{III. $S=1,2$ DIMERIZED HEISENBERG MODELS AND $S=2$ HEISENBERG MODEL WITH UNIAXIAL ISOTROPY}

\section{A. $S=1,2$ dimerized Heisenberg models}

First, we consider $S=1,2$ dimerized Heisenberg models,

$$
H=\sum_{i=1}^{N / 2}\left(J_{1} S_{2 i} \cdot S_{2 i+1}+J_{2} S_{2 i+1} \cdot S_{2 i+2}\right),
$$

where $S_{i}$ is the spin-1 or 2 operators on the $i$ th site and $N$ is the total number of sites. The periodic boundary condition is imposed as $\boldsymbol{S}_{N+i}=\boldsymbol{S}_{i}$ for all of the models in this paper. $J_{1}$ and $J_{2}$ are parametrized as $J_{1}=\sin \theta$ and $J_{2}=\cos \theta$, respectively. We consider the case of $0<\theta<\pi / 2$ in this paper. The ground state is composed of an ensemble of $N / 2$ singlet pairs in limits of $\theta \rightarrow 0$ and $\theta \rightarrow \pi / 2$. The system is equivalent to the isotropic antiferromagnetic Heisenberg chain at $\theta=\pi / 4$. Based on the VBS picture, we expect a reconstruction of the valence bonds by changing $\theta$.

Figures 1(a) and 1(b) show the $\theta$ dependence of the Berry phase on the link with $J_{1}$ coupling and $J_{2}$ coupling with $S=1, N=14$ and $S=2, N=10$, respectively. The region with the Berry phase $\pi$ is shown by the bold line. There are several quantum phase transitions characterized by the Berry phase as the topological order parameters. The boundary of the two regions with different Berry phases 0 and $\pi$ does not have a well-defined Berry phase, since the energy gap closes during the change of the local twist parameter $\phi$. Since the
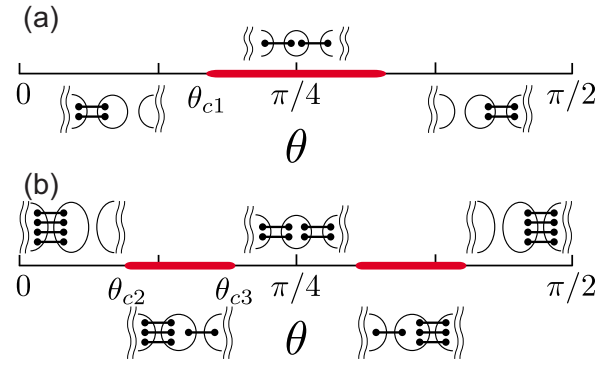

FIG. 1. (Color online) The Berry phases on the local link of (a) the $S=1$ periodic $N=14$ and (b) the $S=2$ periodic $N=10$ dimerized Heisenberg chains. The Berry phase is $\pi$ on the bold line, while that is 0 on the other line. The phase boundaries in the finite size system are $\theta_{c 1}=0.531237, \theta_{c 2}=0.287453$, and $\theta_{c 3}=0.609305$, respectively. The Berry phase in (a) and (b) has an inversion symmetry with respect to $\theta=\pi / 4$. A schematic VBS picture of the ground state is assigned to each phase. Dots, bold lines, and open circles denote the $S=1 / 2$, singlet dimers, and the operations of symmetrization, respectively.

Berry phase is undefined at the boundaries, there exists the level crossing which implies the existence of the gapless excitation in the thermodynamic limit. This result is consistent with the results previously discussed, ${ }^{30}$ which the general integer- $S$ extended string order parameters change as the dimerization changes. The phase diagram defined by our topological order parameter is consistent with the one by the nonlocal string order parameter. In an $N=10$ system with $S=2$, the phase boundaries are $\theta_{c 2}=0.287453$ and $\theta_{c 3}=0.609305$, and it is consistent with the results obtained by using the level spectroscopy which is based on conformal field theory techniques. ${ }^{36}$ Especially, in the one-dimensional case, the energy diagram of the system with twisted link is proportional to that of the system with twisted boundary conditions. However, our analysis focuses on the quantum property of the wave functions rather than the energy diagram.

\section{B. $S=2$ Heisenberg model with uniaxial anisotropy}

As for the $S=2$ Heisenberg model with $D$ term, we use the Hamiltonian

$$
H=\sum_{i}^{N}\left[J \boldsymbol{S}_{i} \cdot \boldsymbol{S}_{i+1}+D\left(S_{i}^{z}\right)^{2}\right] .
$$

Figure 2 shows the Berry phase of the local link in the $S=2$ Heisenberg model $+D$ term with $N=10$. The parameter $J=1$ in our calculations. The region of the bold line has the Berry phase $\pi$, and the other region has the vanishing Berry

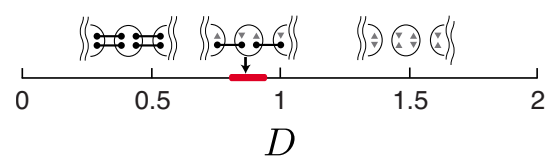

FIG. 2. (Color online) The Berry phases on the local link of the $S=2$ periodic $N=10$ Heisenberg chain with single-ion anisotropy. The notations are the same as in Fig. 1. An up (down) triangle denotes an up (down) spin-1/2. 
phase. This result also makes us possible to consider the Berry phase as a local order parameter of the Haldane spin chains. Our numerical results for finite size systems support the presence of the intermediate $D$ phase. ${ }^{33}$

\section{INTERPRETATION OF THE NUMERICAL RESULTS BY VBS STATE}

Let us now interpret our numerical results in terms of the VBS state picture. The VBS state is the exact ground state of the Affleck-Kennedy-Lieb-Tasaki (AKLT) model. ${ }^{37}$ We shall calculate the Berry phase of the generalized VBS state with the aid of the chiral AKLT model ${ }^{38}$ and its exact ground state wave function. The chiral AKLT model is obtained by applying $\mathrm{O}(2)$ rotation of spin operators in the original AKLT model. In our calculation, it is convenient to introduce the Schwinger boson representation of the spin operators as $S_{i}^{+}=a_{i}^{\dagger} b_{i}, S_{i}^{-}=a_{i} b_{i}^{\dagger}$, and $S_{i}^{z}=\left(a_{i}^{\dagger} a_{i}-b_{i}^{\dagger} b_{i}\right) / 2$. Here, $a_{i}$ and $b_{i}$ satisfy the commutation relation $\left[a_{i}, a_{j}^{\dagger}\right]=\left[b_{i}, b_{j}^{\dagger}\right]=\delta_{i j}$ with all other commutators vanishing. ${ }^{39}$ The constraint $a_{i}^{\dagger} a_{i}+b_{i}^{\dagger} b_{i}=2 S_{i}$ is imposed to reproduce the dimension of the spin $S_{i}$ Hilbert space at each site. In general, the ground state of the chiral AKLT model having $B_{i j}$ valence bonds on the link $\langle i j\rangle$ is written as ${ }^{38}$

$$
\left|\left\{\phi_{i, j}\right\}\right\rangle=\prod_{\langle i j\rangle}\left(e^{i \phi_{i j} / 2} a_{i}^{\dagger} b_{j}^{\dagger}-e^{-i \phi_{i j} / 2} b_{i}^{\dagger} a_{j}^{\dagger}\right)^{B_{i j}}|\mathrm{vac}\rangle .
$$

This state has nonzero average of vector spin chirality $\left\langle\boldsymbol{S}_{i} \times \boldsymbol{S}_{j} \cdot \hat{z}\right\rangle$ unless the twist parameter $\phi_{i j}=0$ or $\pi$. This state is a zero-energy ground state of the following Hamiltonian:

$$
H\left(\left\{\phi_{i, i+1}\right\}\right)=\sum_{i=1}^{N} \sum_{J=J_{\min }}^{J_{\max }} A_{J} P_{i, i+1}^{J}\left[\phi_{i, i+1}\right],
$$

where $\quad J_{\max }=\left(B_{i-1, i}+2 B_{i, i+1}+B_{i+1, i+2}\right) / 2, \quad J_{\min }=\left(B_{i-1, i}\right.$ $\left.+B_{i+1, i+2}\right) / 2+1$, and $A_{J}$ is the arbitrary positive coefficient. $P_{i, i+1}^{J}[0]$ is the polynomial in $\boldsymbol{S}_{i} \cdot \boldsymbol{S}_{i+1}$ and acts as a projection operator projecting the bond spin $\boldsymbol{J}_{i, i+1}=\boldsymbol{S}_{i}+\boldsymbol{S}_{i+1}$ onto the subspace of spin magnitude $J$. The replacement,

$$
S_{i}^{+} S_{i+1}^{-}+S_{i}^{-} S_{i+1}^{+} \rightarrow e^{i \phi_{i, i+1}} S_{i}^{+} S_{i+1}^{-}+e^{-i \phi_{i, i+1}} S_{i}^{-} S_{i+1}^{+},
$$

in $\boldsymbol{S}_{i} \cdot \boldsymbol{S}_{i+1}$ produces $P_{i, i+1}^{J}\left[\phi_{i, i+1}\right]$ in Eq. (6).

Now, we shall explicitly show that the Berry phase of the VBS state extracts the local number of the valence bonds $B_{i j}$ as $B_{i j} \pi(\bmod 2 \pi)$. Let us now consider the local twist of the parameters $\phi_{i j}=\phi \delta_{i j, 12}$ and rewrite the ground state $\left|\left\{\phi_{i, j}\right\}\right\rangle$ as $|\phi\rangle$. To calculate the Berry phase of the VBS state, the following relation is useful:

$$
i \gamma_{12}=i B_{12} \pi+i \int_{0}^{2 \pi} \operatorname{Im}\left[\left\langle\phi\left|\partial_{\phi}\right| \phi\right\rangle\right] / \mathcal{N}(\phi) d \phi,
$$

where $\gamma_{12}$ is the Berry phase of the bond $\langle 12\rangle$ and $\mathcal{N}(\phi)$ $=\langle\phi \mid \phi\rangle$. Note that the first term of the right hand side comes from the gauge fixing of the multivalued wave function to the single-valued function. Then, the only thing to do is to evaluate the imaginary part of the connection.

Let us first consider the $S=1$ VBS state as the simplest example. In this case, $B_{i, i+1}=1$ for any bond and the VBS state with a local twist is given by

$$
|\phi\rangle=\left(e^{i \phi / 2} a_{1}^{\dagger} b_{2}^{\dagger}-e^{-i \phi / 2} b_{1}^{\dagger} a_{2}^{\dagger}\right) \prod_{i=2}^{N}\left(a_{i}^{\dagger} b_{i+1}^{\dagger}-b_{i}^{\dagger} a_{i+1}^{\dagger}\right)|\mathrm{vac}\rangle .
$$

We impose the periodic boundary condition, i.e., $a_{N+1}=a_{1}$ and $b_{N+1}=b_{1}$. It is convenient to introduce the singlet creation operator $s^{\dagger}=\left(a_{1}^{\dagger} b_{2}^{\dagger}-b_{1}^{\dagger} a_{2}^{\dagger}\right)$ and the triplet $\left(J_{z}=0\right)$ creation operator $t^{\dagger}=\left(a_{1}^{\dagger} b_{2}^{\dagger}+b_{1}^{\dagger} a_{2}^{\dagger}\right)$. We can rewrite the bond $\langle 12\rangle$ part of the VBS state $\left(e^{i \phi / 2} a_{1}^{\dagger} b_{2}^{\dagger}-e^{-i \phi / 2} b_{1}^{\dagger} a_{2}^{\dagger}\right)$ as $\left(\cos \frac{\phi}{2} s^{\dagger}\right.$ $\left.+i \sin \frac{\phi}{2} t^{\dagger}\right)$. Then, $|\phi\rangle$ and $\partial_{\phi}|\phi\rangle$ can be written as

$$
\begin{gathered}
|\phi\rangle=\cos \frac{\phi}{2}|0\rangle+i \sin \frac{\phi}{2}|1\rangle, \\
\partial_{\phi}|\phi\rangle=-\frac{1}{2} \sin \frac{\phi}{2}|0\rangle+\frac{i}{2} \cos \frac{\phi}{2}|1\rangle,
\end{gathered}
$$

where

$$
\begin{aligned}
& |0\rangle=s^{\dagger} \prod_{i=2}^{N}\left(a_{i}^{\dagger} b_{i+1}^{\dagger}-b_{i}^{\dagger} a_{i+1}^{\dagger}\right)|\mathrm{vac}\rangle, \\
& |1\rangle=t^{\dagger} \prod_{i=2}^{N}\left(a_{i}^{\dagger} b_{i+1}^{\dagger}-b_{i}^{\dagger} a_{i+1}^{\dagger}\right)|\mathrm{vac}\rangle .
\end{aligned}
$$

It is now obvious that the imaginary part of $\left\langle\phi\left|\partial_{\phi}\right| \phi\right\rangle$ vanishes since the state $|1\rangle$ having a total spin $S_{\text {tot }}=1$ is orthogonal to the state $|0\rangle$ with $S_{\text {tot }}=0$. Therefore, the Berry phase of this state is given by $\gamma_{12}=\pi$. Next, we shall consider a more general situation with arbitrary $B_{i j}$. We can also express the VBS state with a local twist on the bond $\langle 12\rangle$ in terms of $s^{\dagger}$ and $t^{\dagger}$ as

$$
|\phi\rangle=\left(\cos \frac{\phi}{2} s^{\dagger}+i \sin \frac{\phi}{2} t^{\dagger}\right)^{B_{12}} \prod_{i=2}^{N}\left(a_{i}^{\dagger} b_{i+1}^{\dagger}-b_{i}^{\dagger} a_{i+1}^{\dagger}\right)^{B_{i, i+1}}|\mathrm{vac}\rangle .
$$

By using the binomial expansion, $|\phi\rangle$ can be rewritten as

$$
|\phi\rangle=\sum_{k=0}^{B_{12}}\left(\begin{array}{c}
B_{12} \\
k
\end{array}\right)\left(\cos \frac{\phi}{2}\right)^{B_{12}-k}\left(i \sin \frac{\phi}{2}\right)^{k}|k\rangle,
$$

where $|k\rangle=\left(s^{\dagger}\right)^{B_{12}-k}\left(t^{\dagger}\right)^{k}(\cdots)|\mathrm{vac}\rangle$ is the state with $k$ triplet bonds on the link $\langle 12\rangle .(\cdots)$ denotes the rest of the VBS state. In a parallel way,

$$
\begin{aligned}
\partial_{\phi}|\phi\rangle= & \frac{1}{2} \sum_{k=0}^{B_{12}}\left(\begin{array}{c}
B_{12} \\
k
\end{array}\right)\left(\cos \frac{\phi}{2}\right)^{B_{12}-k}\left(i \sin \frac{\phi}{2}\right)^{k} \\
& \times\left(k \cot \frac{\phi}{2}-\left(B_{12}-k\right) \tan \frac{\phi}{2}\right)|k\rangle .
\end{aligned}
$$

To see that the imaginary part of $\left\langle\phi\left|\partial_{\phi}\right| \phi\right\rangle$ is zero, we have to show that $\operatorname{Im}\langle k \mid l\rangle=0$ when $k$ and $l$ have the same parity (even or odd) and $\operatorname{Re}\langle k \mid l\rangle=0$ when $k$ and $l$ have different parities. This can be easily shown by using the coherent state representation of the Schwinger bosons (see the Appendix). Then, using the relation [Eq. (8)], we can obtain the Berry phase as 


$$
\gamma_{i j}=B_{i j} \pi \quad(\bmod 2 \pi) .
$$

This result means that the Berry phase of the generalized VBS state counts the number of the valence bonds on the bond $\langle i j\rangle$. One valence bond has the $\pi$ Berry phase. Finally, it should be stressed that our calculation of the Berry phase is not restricted to one-dimensional VBS states but can be generalized to the VBS state on an arbitrary graph $^{40}$ as long as there is a gap while varying the twist parameter.

Now, let us consider the previous two models in terms of the VBS picture. For the $S=2$ dimerized Heisenberg model, the number of the valence bonds changes as the $\theta$ changes (see Fig. 1). Since the number of the valence bonds on a local link can be computed by the Berry phase, we can clearly see that the reconstruction of the valence bonds occurs during the change of the dimerization. Thus, the result of the Berry phase is consistent with the VBS picture. For the $S=2$ Heisenberg chain with single-ion anisotropy, the valence bonds are broken one by one as $D$ increases, as we can see in Fig. 2. We see that the Berry phase reflects the number of the valence bonds as well as the previous dimerized Heisenberg chain. This can be understood as a fractionalization since the basic objects of the present integer spin chains are spin- $1 / 2$ singlets.

\section{RELATION BETWEEN THE BERRY PHASE AND THE ENTANGLEMENT ENTROPY}

Moreover, the Berry phase of generalized VBS state relates to the number of the edge states which emerge when the spin chain has edges. ${ }^{8}$ Thus, it detects the property of the topological phase. Since the entanglement entropy also detects such phases, ${ }^{13,14,41}$ we clarify the relation between the Berry phase and the entanglement entropy. The entanglement entropy of our generalized VBS state in thermodynamic limit is $\mathcal{S}_{A}=\sum_{(i j) \in \partial A} \log \left(B_{i j}+1\right)$, where $\partial A$ denotes the set of the bonds on the boundary of subsystem $A$. It counts the number of the edge states $g_{\text {edge }}$ as $S_{A}=\log g_{\text {edge }}$. Thus, the Berry phase is related to the entanglement entropy in generalized VBS states via the edge states in the thermodynamic limit. ${ }^{12}$

\section{CONCLUSION}

In conclusion, we have shown that the topological local order parameter defined by quantized Berry phases is useful to classify the phases of various spin chains such as the Haldane phase. In our calculations, the Berry phase is locally defined and does not need nonlocal calculations. It is also useful to estimate the order parameter from the finite size systems since it is quantized even in the finite size systems. The property of the phase is revealed in terms of the texture pattern of the Berry phase. We have also analytically studied the Berry phase of the generalized VBS state and found that the Berry phase picks up the number of singlets on the local link.

\section{ACKNOWLEDGMENTS}

The authors are grateful to I. Maruyama and S. Todo for fruitful discussions. The numerical diagonalization has been accomplished by utilizing the program package TITPACK Version 2. The computation in this work has been done using the facilities of the Supercomputer Center, Institute for Solid State Physics, University of Tokyo. H.K. was supported by the Japan Society for the Promotion of Science. Y.H. was supported by a Grant-in-Aid for Scientific Research on Priority Areas from MEXT (No. 18043007).

\section{APPENDIX}

In this appendix, we show that $\operatorname{Re}\langle k \mid l\rangle=0$ when $k$ and $l$ have different parities and $\operatorname{Im}\langle k \mid l\rangle=0$ when $k$ and $l$ have the same parity by simple symmetry arguments. To show them, it is convenient to introduce a spin coherent state. ${ }^{39}$ For a point $\hat{\Omega}=(\sin \theta \cos \phi, \sin \theta \sin \phi, \cos \theta)$ on the unit sphere, the spin coherent state at each site is defined as

$$
|\hat{\Omega}\rangle=\frac{\left(u a^{\dagger}+v b^{\dagger}\right)^{2 S}}{\sqrt{(2 S) !}}|\mathrm{vac}\rangle
$$

where $(u, v)=\left[\cos (\theta / 2) e^{i \phi / 2}, \sin (\theta / 2) e^{-i \phi / 2}\right]$ are spinor coordinates. Using $|\hat{\Omega}\rangle$, the resolution of the identity is given by

$$
I=\frac{2 S+1}{4 \pi} \int d \hat{\Omega}|\hat{\Omega}\rangle\langle\hat{\Omega}|,
$$

where $I$ denotes a $(2 S+1)$-dimensional identity matrix. Let us now consider the inner product $\langle k \mid l\rangle$. We can set $k \geqslant l$ without loss of generality. Inserting the resolution of the identity [Eq. (A2)] between $\langle k|$ and $|l\rangle$, the integral representation of the inner product can be obtained as

$$
\begin{aligned}
\langle k \mid l\rangle= & \prod_{j=1}^{N}\left(2 S_{j}+1\right) ! \int \prod_{j=1}^{N} \frac{d \hat{\Omega}_{j}}{4 \pi}\left(\frac{1-\hat{\Omega}_{1} \cdot \hat{\Omega}_{2}}{2}\right)^{B_{12}-k} \\
& \times\left(\frac{1+\hat{\Omega}_{1} \cdot \hat{\Omega}_{2}}{2}-\cos \theta_{1} \cos \theta_{2}\right)^{l} K\left(\hat{\Omega}_{1}, \hat{\Omega}_{2}\right)^{k-l} \\
& \times \prod_{i=2}^{N}\left(\frac{1-\hat{\Omega}_{i} \cdot \hat{\Omega}_{i+1}}{2}\right)^{B_{i, i+1}}
\end{aligned}
$$

where

$$
K\left(\hat{\Omega}_{1}, \hat{\Omega}_{2}\right)=\frac{1}{2}\left[\cos \theta_{1}-\cos \theta_{2}-i \sin \theta_{1} \sin \theta_{2} \sin \left(\phi_{1}-\phi_{2}\right)\right]
$$

Here, we have already used the following relation: $\left\langle\operatorname{vac}\left|a^{S-l} b^{S+l}\right| \Omega\right\rangle=\sqrt{(2 S) !} u^{S-l} v^{S+l}$. First, we consider the case where $k$ and $l$ have different parities. In this case, $k-l$ is odd and hence $K\left(\hat{\Omega}_{1}, \hat{\Omega}_{2}\right)^{k-l}$ changes its sign under the change of variables $\left(\theta_{j}, \phi_{j}\right)$ to $\left(\pi-\theta_{j},-\phi_{j}\right)(j=1,2, \ldots, N)$. Since the other part of the integrand is invariant under this change of variables, we obtain $\langle k \mid l\rangle=0$. Therefore, it is now obvious that $\operatorname{Re}\langle k \mid l\rangle=0$ when $k$ and $l$ have different parities. Next, we consider the case where $k$ and $l$ have the same parity. In this case, $k-l$ is even. Thus, we set $k-l=2 m(m \in \mathbf{N})$ and expand $K\left(\hat{\Omega}_{1}, \hat{\Omega}_{2}\right)^{2 m}$ as 


$$
\begin{aligned}
K\left(\hat{\Omega}_{1}, \hat{\Omega}_{2}\right)^{2 m}= & \left(\frac{1}{2}\right)^{2 m} \sum_{n=0}^{2 m}\left(\begin{array}{c}
2 m \\
n
\end{array}\right)\left(\cos \theta_{1}-\cos \theta_{2}\right)^{2 m-n}(-i)^{n} \\
& \times\left[\sin \theta_{1} \sin \theta_{2} \sin \left(\phi_{1}-\phi_{2}\right)\right]^{n} .
\end{aligned}
$$

The imaginary part of $K\left(\hat{\Omega}_{1}, \hat{\Omega}_{2}\right)^{2 m}$ comes from the contribution of the odd $n$ in the above summation. Now, we consider the following change of variables: $\left(\theta_{j}, \phi_{j}\right)$ to $\left(\theta_{j},-\phi_{j}\right)$ $(j=1,2, \ldots, N)$. Under this transformation, $\operatorname{Im}\left[K\left(\hat{\Omega}_{1}, \hat{\Omega}_{2}\right)^{2 m}\right]$ changes its sign. On the other hand, the other part of the integrand in Eq. (A3) is real and invariant under this change of variables. Therefore, $\operatorname{Im}\langle k \mid l\rangle=0$ when $k$ and $l$ have the same parity. Finally, we remark that the generalization of the above result to the VBS state on an arbitrary graph is almost trivial since we have not used a specific property of the onedimensional VBS state in our proof. *hirano@pothos.t.u-tokyo.ac.jp

†katsura@ appi.t.u-tokyo.ac.jp

thatsugai@sakura.cc.tsukuba.ac.jp

${ }^{1}$ Y. Hatsugai, J. Phys. Soc. Jpn. 75, 123601 (2006).

${ }^{2}$ X. G. Wen, Phys. Rev. B 40, 7387 (1989).

${ }^{3}$ Y. Hatsugai, J. Phys. Soc. Jpn. 73, 2604 (2004).

${ }^{4}$ Y. Hatsugai, J. Phys. Soc. Jpn. 74, 1374 (2005).

${ }^{5}$ R. B. Laughlin, Phys. Rev. B 23, 5632 (1981).

${ }^{6}$ Y. Hatsugai, Phys. Rev. Lett. 71, 3697 (1993).

${ }^{7}$ Y. Hatsugai, Phys. Rev. B 48, 11851 (1993).

${ }^{8}$ T. Kennedy, J. Phys.: Condens. Matter 2, 5737 (1990).

${ }^{9}$ M. Hagiwara, K. Katsumata, I. Affleck, B. I. Halperin, and J. P. Renard, Phys. Rev. Lett. 65, 3181 (1990).

${ }^{10}$ W. P. Su, J. R. Schrieffer, and A. J. Heeger, Phys. Rev. Lett. 42, 1698 (1979).

${ }^{11}$ N. Read and S. Sachdev, Phys. Rev. Lett. 62, 1694 (1989).

${ }^{12}$ S. Ryu and Y. Hatsugai, Phys. Rev. B 73, 245115 (2006).

${ }^{13}$ T. Hirano and Y. Hatsugai, J. Phys. Soc. Jpn. 76, 074603 (2007).

${ }^{14}$ H. Katsura, T. Hirano, and Y. Hatsugai, Phys. Rev. B 76, 012401 (2007).

15 A. Kitaev and J. Preskill, Phys. Rev. Lett. 96, 110404 (2006).

${ }^{16}$ M. Levin and X. G. Wen, Phys. Rev. Lett. 96, 110405 (2006).

${ }^{17}$ M. V. Berry, Proc. R. Soc. London, Ser. A 392, 45 (1984).

${ }^{18}$ I. Maruyama and Y. Hatsugai, J. Phys. Soc. Jpn. 76, 113601 (2007).

${ }^{19}$ Y. Hatsugai, J. Phys.: Condens. Matter 19, 145209 (2007).

${ }^{20}$ F. D. M. Haldane, Phys. Lett. 93A, 464 (1983).

${ }^{21}$ Y. Ajiro, T. Goto, H. Kikuchi, T. Sakakibara, and T. Inami, Phys. Rev. Lett. 63, 1424 (1989).

${ }^{22}$ G. E. Granroth, M. W. Meisel, M. Chaparala, T. Jolicoeur, B. H.
Ward, and D. R. Talham, Phys. Rev. Lett. 77, 1616 (1996).

${ }^{23}$ I. Affleck, J. Phys.: Condens. Matter 19, 3047 (1989).

${ }^{24}$ T. Kennedy and H. Tasaki, Phys. Rev. B 45, 304 (1992).

${ }^{25}$ T. Kennedy and H. Tasaki, Commun. Math. Phys. 147, 431 (1992).

${ }^{26}$ M. den Nijs and K. Rommelse, Phys. Rev. B 40, 4709 (1989).

${ }^{27}$ M. Nakamura and S. Todo, J. Phys. Soc. Jpn. 145, 217 (2002).

${ }^{28}$ M. Nakamura and S. Todo, Phys. Rev. Lett. 89, 077204 (2002).

${ }^{29}$ K. Hida, Phys. Rev. B 45, 2207 (1992).

${ }^{30}$ M. Yamanaka, M. Oshikawa, and S. Miyashita, J. Phys. Soc. Jpn. 65, 1562 (1996).

${ }^{31}$ F. D. M. Haldane, Phys. Rev. Lett. 50, 1153 (1983).

${ }^{32}$ H. Aschauer and U. Schollwöck, Phys. Rev. B 58, 359 (1998).

${ }^{33}$ M. Oshikawa, J. Phys.: Condens. Matter 4, 7469 (1992).

${ }^{34}$ M. Oshikawa, M. Yamanaka, and S. Miyashita, arXiv:cond-mat/ 9507098 (unpublished).

${ }^{35}$ T. Fukui, Y. Hatsugai, and H. Suzuki, J. Phys. Soc. Jpn. 74, 1674 (2006).

${ }^{36}$ A. Kitazawa and K. Nomura, J. Phys. Soc. Jpn. 66, 3379 (1997).

${ }^{37}$ I. Affleck, T. Kennedy, E. H. Lieb, and H. Tasaki, Phys. Rev. Lett. 59, 799 (1987).

${ }^{38}$ R. Dillenschneider, J. H. Kim, and J. H. Han, arXiv:0705.3993 (unpublished).

${ }^{39} \mathrm{~A}$. Auerbach, Interacting Electrons and Quantum Magnetism (Springer, New York, 1998).

${ }^{40}$ A. N. Kirillov and V. E. Korepin, St. Petersbg. Math. J. 1, 47 (1990).

${ }^{41}$ H. Fan, V. Korepin, and V. Roychowdhury, Phys. Rev. Lett. 93, 227203 (2004) 